\title{
Acknowledgment of Reviewers, 2011
}

The success of Phytopathology depends on the quality of manuscripts submitted by authors and on the care and competence with which they are reviewed. It is the policy of the Editorial Board to solicit reviews for manuscripts from specialists who are most qualified to review them. In addition to members of the Editorial Board, the individuals listed below provide constructive critical reviews of one or more manuscripts during the past year. Their names are published here in grateful appreciation of their contribution to the journal and to the science of phytopathology. http://dx.doi.org/10.1094/PHYTO-102-1-0004

Abdo, Z., University of Idaho, Moscow, ID, U.S.A.

Acevedo, M., North Dakota State University, Fargo, ND, U.S.A.

Adams, B., Brigham Young University, Provo, UT, U.S.A.

Adaskaveg, J. E., University of California, Riverside, CA, U.S.A.

Adkins, S., U.S. Horticultural Research Laboratory, Fort Pierce, FL,

U.S.A.

Allen, C., University of Wisconsin, Madison, WI, U.S.A.

Allen, T. W., Mississippi State University, Stoneville, MS, U.S.A.

Almeida, R., University of California, Berkeley, CA, U.S.A.

Anderson, J., University of Toronto, Mississauga, Ontario, CANADA

Anke, M., University of Southern Queensland, Toowoomba, Queensland,

AUSTRALIA

Ashikawa, I., NARO Institute of Crop Science, Tsukuba, Ibaraki, JAPAN

Bacon, C., USDA-ARS, Athens, GA, U.S.A.

Bai, G., Kansas State University at Manhattan, Manhattan, KS, U.S.A.

Bailey, B., Agricultural Research Service, Beltsville, MD, U.S.A.

Bakker, M., University of Minnesota, St. Paul, MN, U.S.A.

Bakkeren, G., Agricultur \& Agri-Food Canada, Summerland, British

Columbia, CANADA

Balint-Kurti, P. J., USDA-ARS, Raleigh, NC, U.S.A.

Barak, J., University of Wisconsin, Madison, WI, U.S.A.

Bariana, H., University of Sydney, Narellan, New South Wales,

AUSTRALIA

Barnes, I., Forestry and Agricultural Biotechnology Institute (FABI),

Pretoria, Gauteng, SOUTH AFRICA

Beal, C., University of Texas at Austin, Austin, TX, U.S.A.

Beckerman, J., Purdue University, West Lafayette, IN, U.S.A.

Belanger, R., Laval University, Quebec, Quebec, CANADA

Bennett, R. S., USDA-ARS, Shafter, CA, U.S.A.

Biggs, A. R., West Virginia University, Kearneysville, WV, U.S.A.

Bolton, M. D., U.S. Department of Agriculture, St. Paul, MN, U.S.A.

Boonham, N., Food and Environment Research Agency, Sand Hutton,

York, UNITED KINGDOM

Boopathi Narayanan, M., Tamil Nadu Agricultural University,

Coimbatore, Tamil Nadu, INDIA

Borneman, J., University of California, Riverside, CA, U.S.A.

Bosland, P. W., New Mexico State University, Las Cruces, NM, U.S.A.

Bostock, R. M., University of California, Davis, CA, U.S.A.

Brasier, C., Forest Research, Farnham, Surrey, UNITED KINGDOM

Brodhagen, M., USDA, Corvallis, OR, U.S.A.

Brown, C. R., USDA-ARS, Prosser, WA, U.S.A.

Brueggeman, R. S., North Dakota State University, Fargo, ND, U.S.A.

Budge, G. E., Central Science Laboratory, York, N Yorks, UNITED

KINGDOM

Bull, C. T., USDA-ARS, Salinas, CA, U.S.A.

Burlakoti, R. R., Weather INnovations Incorporated, Chatham, Ontario, CANADA

Burpee, L., University of Georgia, Griffin, GA, U.S.A.

Busman, M., USDA-ARS, Peoria, IL, U.S.A.

Byamukama, E., University of Nebraska, Lincoln, NE, U.S.A.

Cadle-Davidson, L., USDA-ARS, GGRU, Geneva, NY, U.S.A.

Cafe-Filho, A. C., Universidade de Brasilia, Brasilia, DF, BRAZIL

Cai, G., Cornell Univeristy, Ithaca, NY, U.S.A.

Carisse, O., Agriculture and AgriFood Canada, St-Jean-sur-Richelieu,

Quebec, CANADA

Carson, M., USDA-ARS, St. Paul, MN, U.S.A.

Carter, L., Syngenta Seeds, Inc., Nampa, ID, U.S.A.

Ceresini, P., UNESP University of São Paulo State, Ilha Solteira Campus,

Ilha Solteira, São Paulo, BRAZIL

Chakraborty, S., CSIRO, Brisbane, Queensland, AUSTRALIA

Chastagner, G. A., Washington State University, Puyallup, WA, U.S.A.
Chen, X., USDA-ARS and Washington State University, Pullman, WA, U.S.A.

Chilvers, M. I., Michigan State University, East Lansing, MI, U.S.A.

Chitrampalam, P., University of California, Davis, Salinas, CA, U.S.A.

Chitwood, D., USDA-ARS, Beltsville, MD, U.S.A.

Chung, K., University of Florida, Lake Alfred, FL, U.S.A.

Clarkson, J. P., University of Warwick, Warwick, Warwickshire,

UNITED KINGDOM

Conner, K., Auburn University, AL, U.S.A.

Cooke, L. R., Queen's University of Belfast, Belfast, Northern Ireland, UNITED KINGDOM

Cooper, B., USDA-ARS, Beltsville, MD, U.S.A.

Coplin, D., The Ohio State University, Columbus, OH, U.S.A.

Correll, J. C., University of Arkansas, Fayetteville, AR, U.S.A.

Cowger, C., USDA-ARS, Raleigh, NC, U.S.A.

Cox, K. D., Cornell University, Geneva, NY, U.S.A.

Craven, K., Samuel Roberts Noble Foundation, Ardmore, OK, U.S.A.

da Silva, A., Universidade de Sao Paulo, Sao Paulo, BRAZIL

Damann, K. E., Louisiana State University Agricultural Center, Baton

Rouge, LA, U.S.A.

De Cal, A., INIA, Madrid, SPAIN

De Koeyer, D., Agriculture and Agri-Food Canada, Fredericton, New

Brunswick, CANADA

Del Ponte, E. M., Iowa State University, Ames, IA, U.S.A.

Del Ponte, E., Universidade Federal do Rio Grande do Sul, Porto Alegre, Rio Grande do Sul, BRAZIL

DeMarsay, A., Centreville, VA, U.S.A.

Denny, T., Unversity of Georgia, Athens, GA, U.S.A.

Deom, M., University of Georgia, Athens, GA, U.S.A.

Dewdney, M. M., University of Florida, Lake Alfred, FL, U.S.A.

Dhillon, B., University of British Columbia, Vancouver, British

Columbia, CANADA

Díaz-Mínguez, J. M., University of Salamanca, Villamayor, Salamanca, SPAIN

Ding, B., Ohio State University, Columbus, OH, U.S.A.

Domier, L., USDA-ARS, Urbana, IL, U.S.A.

Donald, C., Department of Primary Industries Victoria, Ferntree Gully

Delivery Centre, Victoria, AUSTRALIA

Dugan, F., USDA-ARS, Pullman, WA, U.S.A.

Dunigan, D., University of Nebraska-Lincoln, Lincoln, NE, U.S.A.

Eid, S., Moscow, ID, U.S.A.

Esker, P., University of Wisconsin, Madison, WI, U.S.A.

Fakhoury, A. M., Southern Illinois University, Carbondale, IL, U.S.A.

Falk, B., University of California, Davis, CA, U.S.A.

Fellers, J., USDA-ARS, Manhattan, KS, U.S.A.

Feng, J., Crop Diversification Centre North, Edmonton, Alberta, CANADA

Fernando, D. G., University of Manitoba, Winnipeg, Manitoba, CANADA

Ferrandino, F. J., The Connecticut Agricultural Experiment Station, New Haven, CT, U.S.A.

Figueira, A., Universidade de São Paulo, Piracicaba, São Paulo, BRAZIL

Fitt, B. D., Rothamsted Research, Harpenden, Hertfordshire, UNITED KINGDOM

Flores, R., Instituto de Biología Molecular y Celular de Plantas (UPVCSIC), Valencua, SPAIN

Folimonova, S. Y., University of Florida, Lake Alfred, FL, U.S.A.

French, R., USDA-ARS, Lincoln, NE, U.S.A.

Fry, W., Cornell University, Ithaca, NY, U.S.A.

Fuchs, M., Cornell University, Geneva, NY, U.S.A.

Fulbright, D. W., Michigan State University, East Lansing, MI, U.S.A. 
Gadoury, D., Cornell University, NYSAES, Geneva, NY, U.S.A.

Garbelotto, M., University of California, Berkeley, CA, U.S.A.

Garcia-Arenal, F., Universidad Politecnica de Madrid, Madrid, SPAIN

Georgi, L., Rutgers University, Chatsworth, NJ, U.S.A.

Gilbertson, R., University of California, Davis, CA, U.S.A.

Gordon, T., Corvallis, OR, U.S.A.

Goss, E. M., University of Florida, Gainesville, FL, U.S.A.

Grewal, T., Saskatchewan Research Council, Saskatoon, Saskatchewan, CANADA

Grunwald, N. J., USDA-ARS, Corvallis, OR, U.S.A.

Gubler, W., University of California, Davis, CA, U.S.A.

Gudmestad, N. C., North Dakota State University, Fargo, ND, U.S.A.

Guttman, D. S., University of Toronto, Toronto, Ontario, CANADA

Hammerschmidt, R., Michigan State University, East Lansing, MI,

U.S.A.

Hammond, R., USDA-ARS, Beltsville, MD, U.S.A.

Hansen, E., Oregon State University, Corvallis, OR, U.S.A.

Hanson, L., USDA-ARS, East Lansing, MI, U.S.A.

Harris, M., Iowa State University, Ames, IA, U.S.A.

Haudenshield, J., University of Illinois, Urbana, IL, U.S.A.

Hausbeck, M. K., Michigan State University, East Lansing, MI, U.S.A.

Hillman, B., Rutgers University, New Brunswick, NJ, U.S.A.

Hsiang, T., University of Guelph, Guelph, Ontario, CANADA

Jacobs, J., Michigan State University, E. Lansing, MI, U.S.A.

Jenkins, D. M., University of Hawaii, Honolulu, HI, U.S.A.

Johnson, D. A., Washington State University, Pullman, WA, U.S.A.

Joly, D. L., Agriculture and Agri-Food Canada, Summerland, British

Columbia, CANADA

Jones, J. B., University of Florida, Gainesville, FL, U.S.A.

Kasarda, D., USDA, Albany, CA, U.S.A.

Kaye, A., Raleigh, NC, U.S.A.

Keinath, A., Clemson University, Charleston, SC, U.S.A.

Kelly, J., Michigan State University, East Lansing, MI, U.S.A.

Kennelly, M. M., Kansas State University, Manhattan, KS, U.S.A.

Kirkpatrick, B., University of California, Davis, CA, U.S.A.

Klosterman, S. J., USDA-ARS, Salinas, CA, U.S.A.

Kobayashi, D. Y., Rutgers University, New Brunswick, NJ, U.S.A.

Kohn, L., University of Toronto, Mississauga, Ontario, CANADA

Kotasthane, A. S., Indira Gandhi Krishi Vishwavidyalaya, Raipur,

Chhattisgarh, INDIA

Kousik, S., U.S. Vegetable Laboratory, Charleston, SC, U.S.A.

Kovacs, L., Missouri State University, Springfield, MO, U.S.A.

Kriss, A. B., The Ohio State University, Wooster, OH, U.S.A.

Kung, S. H., UC Berkeley, Berkeley, CA, U.S.A.

LaMondia, J., The Connecticut Agricultural Experiment Station,

Windsor, CT, U.S.A.

Lamour, K., University of Tennessee, Knoxville, TN, U.S.A.

Larkin, R., USDA-ARS, Orono, ME, U.S.A.

Lazarovits, G., A\&L Biologicals, London, Ontario, CANADA

Lefort, F., University of Applied Sciences of Western Switzerland, Jussy,

Geneva, SWITZERLAND

Leslie, J. F., Kansas State University, Manhattan, KS, U.S.A.

Lewis, K. J., UNBC, Prince George, British Columbia, CANADA

Li, W., USDA-APHIS, Beltsville, MD, U.S.A.

Lindeberg, M., Cornell University, Ithaca, NY, U.S.A.

Liu, M., Cereal Disease Lab, St. Paul, MN, U.S.A.

Loper, J., USDA-ARS, Corvallis, OR, U.S.A.

López, M. M., I.V.I.A., Moncada, Valencia, SPAIN

Lu, H., UFL, Belle Glade, FL, U.S.A.

Luo, Y., University of California, Kearney Agricultural Center, Parlier,

CA, U.S.A.

Luster, D., USDA-ARS, Ft. Detrick, MD, U.S.A.

Madden, L. V., Ohio State University, Wooster, OH, U.S.A.

Manners, J., CSIRO, Brisbane, Queensland, AUSTRALIA

Marra, R. E., Connecticut Agricultural Experiment Station, New Haven,

CT, U.S.A.

Marsolais, F., Agriculture and Agri-Food Canada, London, Ontario,

CANADA

Martin, F. N., USDA-ARS, Salinas, CA, U.S.A.

Martin, R., USDA-ARS-HCRL, Corvallis, OR, U.S.A.

Martyn, R., Purdue University, West Lafayette, IN, U.S.A.

Maurhofer, M., Swiss Federal Institute of Technology Zürich, Zürich,

ZH, SWITZERLAND

Mavrodieva, V. A., USDA-APHIS PPQ-CPHST National Plant

Germplasm and Biotechnology Laboratory, Beltsville, MD, U.S.A.
Mbofung, G., Iowa State University, Ames, IA, U.S.A.

McCallum, B., Agriculture and Agri-Food Canada, Winnipeg, Manitoba, CANADA

McCartney, C., University of Saskatchewan, Saskatoon, Saskatchewan, CANADA

McGhee, G., Michigan State University, East Lansing, MI, U.S.A.

McInroy, J. A., Auburn University, Auburn University, AL, U.S.A.

McSpadden Gardener, B. B., Ohio State University-OARDC, Wooster,

OH, U.S.A.

Melouk, H., USDA-ARS, Stillwater, OK, U.S.A.

Michailides, T., University of California, Kearney Agricultural Center, Parlier, CA, U.S.A.

Miklas, P., Prosser, WA, U.S.A.

Milgroom, M. G., Cornell University, Ithaca, NY, U.S.A.

Miller, L., University of Missouri, Columbia, MO, U.S.A.

Milus, G., University of Arkansas, Fayetteville, AK, U.S.A.

Mitkowski, N., University of Rhode Island, Kingston, RI, U.S.A.

Mondego, J., Instituto Agronômico de Campinas, Campinas, BR|SP, BRAZIL

Mongkolporn, O., Kasetsart University, Kamphaeng Saen, Nakhon Pathom, THAILAND

Montesinos, E., University of Girona, Girona, GIRONA, SPAIN

Munkvold, G., Iowa State University, Ames, IA, U.S.A.

Murphy, J. F., Auburn University, AL, U.S.A.

Myers, A., Iowa State University, Ames, IA, U.S.A.

Navabi, A., University of Guelph, Guelph, Ontario, CANADA

Navas-Cortés, J. A., Institute for Sustainable Agriculture-CSIC, Cordoba, SPAIN

Nelson, E. B., Cornell University, Ithaca, NY, U.S.A.

Nelson, R. S., Samuel Roberts Noble Foundation, Inc., Ardmore, OK, U.S.A.

Nemchinov, L. G., USDA-ARS, Beltsville, MD, U.S.A.

Ngugi, H. K., Penn State University, Biglerville, PA, U.S.A.

Nita, M., Virginia Tech, Winchester, VA, U.S.A.

Nolte, P., University of Idaho, Idaho Falls, ID, U.S.A.

Norelli, J. L., USDA-ARS, Kearneysville, WV, U.S.A.

Norman, D. J., University of Florida, MREC, Apopka, FL, U.S.A.

Nunney, L., University of California, Riverside, Riverside, CA, U.S.A.

O'Donnell, K., USDA-ARS-NCAUR, Peoria, IL, U.S.A.

Ojiambo, P. S., North Carolina State University, Raleigh, NC, U.S.A.

Oliver, R. P., Murdoch University, Perth, Western Australia, AUSTRALIA

Oudemans, P. V., Rutgers University, Chatsworth, NJ, U.S.A.

Ownley, B. H., University of Tennessee, Knoxville, TN, U.S.A.

Ozkilinc, H., Washington State University, Pullman, WA, U.S.A.

Padmanabhan, M., UC Davis, Davis, CA, U.S.A.

Palomares Rius, J., Scottish Crop Research Institute-Invergowrie,

Invergowrie, Dundee, Scotland, UNITED KINGDOM

Palumbo, J. D., USDA-ARS, Albany, CA, U.S.A.

Pastor-Corrales, M. A., USDA-ARS, Beltsville, MD, U.S.A.

Paul, P. A., Ohio State University, Wooster, OH, U.S.A.

Pedley, K. F., USDA-ARS, Ft. Detrick, MD, U.S.A.

Peres, N., University of Florida, Wimauma, FL, U.S.A.

Pettis, G., Louisiana State University, Baton Rouge, LA, U.S.A.

Pfender, W., Corvallis, OR, U.S.A.

Philion, V., IRDA, Saint-Bruno-de-Montarville, Quebec, CANADA

Polston, J. E., University of Florida, Gainesville, FL, U.S.A.

Polverari, A., Università di Verona, Verona, VT, ITALY

Raposo, R., INIA, Madrid, SPAIN

Redinbaugh, M., USDA-ARS Corn and Soybean Research, Wooster, OH, U.S.A.

Rezzonico, F., ACW Waedenswil, Waedenswil, ZH, SWITZERLAND

Rioux, D., NRCan, Québec, CANADA

Rizzo, D. M., University of California, Davis, CA, U.S.A.

Roberts, P., University of Florida, Immokalee, FL, U.S.A.

Robertson, N. L., USDA, Palmer, AL, U.S.A.

Roper, M., UC Berkeley, Berkeley, CA, U.S.A.

Rosenberger, D., Cornell University/NYAES, Highland, NY, U.S.A.

Rowhani, A., UC Davis, Davis, CA, U.S.A.

Rush, C. M., TAES, Bushland, TX, U.S.A.

Sabanadzovic, S., Mississippi State University, MS, U.S.A.

Sands, D., Montana State Univeristy, Bozeman, MT, U.S.A.

Sanjaya, G., Agriculture and Agri-Food Canada, Saskatoon,

Saskatchewan, CANADA

Schaafsma, A., University of Guelph, Ridgetown, Ontario, CANADA 
Scherm, H., University of Georgia, Athens, GA, U.S.A.

Schmolke, M., Technische Universitaet Muenchen, Freising, Bavaria, GERMANY

Schnabel, G., Clemson University, Clemson, SC, U.S.A.

Schneider, R. W., Louisiana State University, Baton Rouge, LA, U.S.A.

Scofield, S., USDA-ARS, West Lafayette, IN, U.S.A.

Scott, J., University of Florida, Gulf Coast Research and Education

Center, Wimauma, FL, U.S.A.

Secor, G. A., North Dakota State University, Fargo, ND, U.S.A.

Shah, D. A., Lewiston, NY, U.S.A.

Sharma, A., GBPUAT, US Nagar, Uttarakhand, INDIA

Sherwood, J., University of Georgia, Athens, GA, U.S.A.

Shew, B., North Carolina State University, Raleigh, NC, U.S.A.

Shipp, L., Agriculture and Agri-Food Canada, Harrow, Ontario, CANADA

Shishkoff, N., USDA-ARS, Frederick, MD, U.S.A

Singh, M., ACS Inc., Fredericton, New Brunswick, CANADA

Singh, P. K., North Dakota State University, Fargo, ND, U.S.A.

Singh, U., International Rice Research Institute, New Delhi, Delhi, INDIA

Sipes, B., University of Hawaii, Honolulu, HI, U.S.A.

Smart, C. D., Cornell University, Geneva, NY, U.S.A.

Solomon, P., The Australian National University, Canberra, Australian Capital Territory, AUSTRALIA

Starr, J., Texas A\&M University, College Station, TX, U.S.A.

Stefani, F., Université Laval, Québec, CANADA

Stenger, D. C., USDA-ARS, Parlier, CA, U.S.A.

Stewart, J., Pullman, WA, U.S.A.

Stotz, H., Oregon State University, Corvallis, OR, U.S.A.

Strelkov, S., University of Alberta, Edmonton, Alberta, CANADA

Subbarao, K. V., University of California, Davis, Salinas, CA, U.S.A.

Sudarshana, M., UC Davis, Davis, CA, U.S.A.

Sutton, T. B., North Carolina State Universtiy, Raleigh, NC, U.S.A.

Talbert, L., Montana State University, Bozeman, MT, U.S.A.

Tatineni, S., USDA-ARS, Lincoln, NE, U.S.A.

Terauchi, R., Iwate Biotechnology Research Center, Kitakami, Iwate, JAPAN

Tian, B., New Haven, CT, U.S.A.

Timper, P., USDA-ARS, Tifton, GA, U.S.A.

Torzilli, A., George Mason University, Fairfax, VA, U.S.A.
Tredway, L. P., North Carolina State University, Raleigh, NC, U.S.A.

Triplett, L., Colorado State University, Fort Collins, CO, U.S.A.

Tyler, B., Virginia Polytechnic Institute and State University, Blacksburg, VA, U.S.A.

Uehling, J., Humboldt State University, Arcata, CA, U.S.A.

Vaillancourt, L. J., University of Kentucky, Lexington, KY, U.S.A.

Vallad, G. E., University of Florida, Wimauma, FL, U.S.A.

van Bruggen, A. H., University of Florida, Gainesville, FL, U.S.A.

Venette, R., USDA Forest Service, St. Paul, MN, U.S.A

Wallwork, H., South Australian Research and Development Institute, Adelaide, South Australia, AUSTRALIA

Wang, D., Michigan State University, East Lansing, MI, U.S.A.

Wang, N., University of Florida, Lake Alfred, FL, U.S.A.

Wayadande, A. C., Oklahoma State University, Stillwater, OK, U.S.A.

Webb, K., USDA-ARS, Ft. Collins, CO, U.S.A.

Webster, C., USHRL-USDA-ARS, Fort Pierce, FL, U.S.A.

Weiland, J., USDA-ARS, Corvallis, OR, U.S.A.

Weller, D. M., USDA-ARS, Washington State University, Pullman, WA, U.S.A.

Whitfield, A. E., Kansas State University, Manhattan, KS, U.S.A.

Whitworth, J., USDA-ARS, Aberdeen, ID, U.S.A.

Willocquet, L., International Rice Research Institute (IRRI), Metro Manila, PHILIPPINES

Wilson, C. R., University of Tasmania, Hobart, Tasmania, AUSTRALIA

Wintermantel, W. M., USDA-ARS, Salinas, CA, U.S.A.

Wong, F. P., University of California, Riverside, CA, U.S.A.

Woodward, S., University of Aberdeen, Aberdeen, Scotland, UNITED KINGDOM

Wu, D., Cornell University, Ithaca, NY, U.S.A.

Xia, X., Chinese Academy of Agricultural Sciences, Beijing, CHINA

Xiao, C., Washington State University, Wenatchee, WA, U.S.A.

Xiong, Z., University of Arizona, Tucson, AZ, U.S.A.

Yajima, W., NDSU, Fargo, ND, U.S.A.

Yakabe, L., USDA-ARS, Davis, CA, U.S.A.

Yan, S., Duke University, Durham, NC, U.S.A.

Yin, C., Washington State University, Pullman, WA, U.S.A.

Zasada, I. A., USDA-ARS, Corvallis, OR, U.S.A.

Zhang, S., University of Kentucky, Lexington, KY, U.S.A.

Zhang, S., University of Florida, Gainesville, FL, U.S.A.

Zhou, S., Texas A\&M University System, Beaumont, TX, U.S.A. 